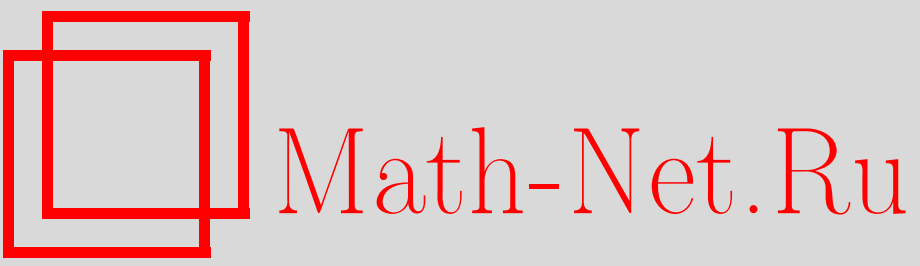

А. В. Архангельский, О свойствах топологических разбиений и об отображениях топологических групп, Итоги науки и техн. Сер. Соврем. мат. и ее прил. Темат. обз., 2020, том 179, 3-9

DOI: https://doi.org/10.36535/0233-6723-2020-179-3-9

Использование Общероссийского математического портала Math-Net.Ru подразумевает, что вы прочитали и согласны с пользовательским соглашением

http://www.mathnet.ru/rus/agreement

Параметры загрузки:

IP : 54.197 .130 .99

26 апреля 2023 г., 18:17:08 


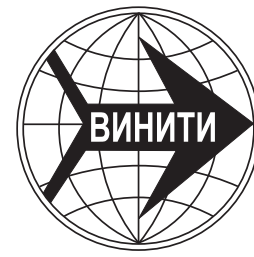

ИТОГИ НАУКИ И ТЕХНИКИ.

Современная математика и ее приложения.

Тематические обзоры.

Том 179 (2020). C. 3-9

DOI: $10.36535 /$ 0233-6723-2020-179-3-9

УДК $512.54,515.12$

\title{
О СВОЙСТВАХ ТОПОЛОГИЧЕСКИХ РАЗБИЕНИЙ И ОБ ОТОБРАЖЕНИЯХ ТОПОЛОГИЧЕСКИХ ГРУПП
}

\author{
(c) 2020 г. $\quad$ А. В. АРХАНГЕЛЬСКИЙ
}

\begin{abstract}
АннотАция. В статье изучаются топологические разбиения топологических пространств, возникающие в связи с непрерывными отображениями топологических пространств. Содержание статьи теснейшим образом связано с такими классическими фундаментальными понятиями общей топологии, как компактность, однородность и полнота по Чеху. Получены новые факты, относящиеся к указанным понятиям.
\end{abstract}

Ключевые слова: топологическое разбиение, полное по Чеху пространство, $k$-накрывающее отображение, диадический компакт, фактор-топология, топологическая группа.

\section{PROPERTIES OF TOPOLOGICAL PARTITIONS AND MAPPINGS OF TOPOLOGICAL GROUPS}

\author{
(c) 2020 A. V. ARKHANGEL'SKII
}

\begin{abstract}
In this paper, we study topological partitions of topological spaces that arise in connection with continuous mappings of topological spaces. The content of the paper is closely related to such classical fundamental concepts of general topology as compactness, homogeneity, and Čech completeness. New facts related to these concepts are obtained.
\end{abstract}

Keywords and phrases: topological partition, Čech complete space, $k$-covering mapping, dyadic compactum, factor topology, topological group.

AMS Subject Classification: 54A25, 54B05

1. Введение. Как в теории общих топологических пространств (называемой также общей, или теоретико-множественной, топологией), так и в топологичесой алгебре естественно (и неизбежно) возникают математические объекты несколько более общей природы, чем топологические пространства - топологические разбиения. Возникли они давно, и прежде всего на основе понятия непрерывного отображения, играющего основную роль при сравнении топологических пространств. Представляется, что топологические разбиения будут играть в дальнейшем все большую роль в теории топологических пространств и в топологической алгебре. Ниже мы делаем отдельные шаги в этом направлении, вводя ряд понятий, относящихся непосредственно к топологическим разбиениям, и намечаем в связи с этим новые вопросы. В то же время содержание статьи теснейшим образом связано с такими классическими фундаментальными понятиями общей топологии, как компактность, однородность и полнота по Чеху. Получены новые факты, относящиеся к указанным понятиям.

В целом используемые обозначения и терминология такие же, как в $[11,14]$. Напомним однако, что теснота топологического пространства $X$ счетна, если для каждого множества $A \subset X$ и каждой точки $x$ из замыкания множества $A$ найдется счетное подмножество $B$ множества $A$, содержащее точку $x$ в своем замыкании. Мощность множества $X$ обозначается $|X|$. Если явно 
не указано предположение об отделимости в пространстве, то это пространство следует считать хаусдорфовым. Тихоновское пространство $X$ полно по Чеху, если оно является множеством типа $G_{\delta}$ в некотором своем компактном хаусдорфовом расширении. Напомним также, что непрерывное отображение $f$ пространства $X$ на пространство $Y$ называется $k$-накрывающим, или компактно накрывающим, если для каждого компакта $F$, лежащего в $Y$, найдется компакт $K$, лежащий в $X$, такой что $f(K)=F$ (см. [1,3]. Здесь и ниже мы называем компактами компактные хаусдорфовы пространства.

Весьма естественно топологические разбиения возникают в топологической алгебре. Напомним, что группа $G$ с топологией $\mathcal{T}$ называется левой топологической группой, если левый сдвиг $l_{x}: G \rightarrow G$, заданный формулой $l_{x}(y)=x y$ для каждого $y \in G$, является гомеоморфизмом пространства $(G, \mathcal{T})$ на себя при каждом $x$ из $G$. Более общие примеры топологических разбиений связаны с произвольными непрерывными отображениями.

Тематика данной статьи относится к тому направлению топологической алгебры, к которому принадлежат ставшие уже классическими работы В. В. Успенского $[7,8]$, М. М. Чобана $[9,10]$, Э. Майкла $[15,16]$ и Яна ван Милла [17] (см. также $[4,5]$. Диадические компакты - это непрерывные образы канторовых кубов $D^{\tau}$, где $D$-дискретное двоеточие. Компакт $X$ называется компактом Дугунджи, если для каждого нульмерного компакта $Z$ и его замкнутого подмножества $A$ всякое непрерывное отображение $f$ пространства $A$ в $X$ продолжается до непрерывного отображения компакта $Z$ в компакт $X$ (см. $[7,8])$.

$\mathrm{K}$ числу основных результатов статьи относятся, в частности, теоремы 4.3, 4.4, 4.9, 4.11 и следствие 4.6. Они касаются свойств компактов, являющихся непрерывными образами топологических групп при непрерывных замкнутых или открытых отображениях.

2. Некоторые новые понятия и предварительные замечания. Разбиением множества $X$ или топологического пространства $(X, \mathcal{T})$ называется покрытие $\gamma$ множества $X$ непустыми попарно не пересекающимися его подмножествами. Топологическим разбиением топологического пространства $(X, \mathcal{T})$ назовем пару $[(X, \mathcal{T}), \gamma]$, где $\gamma$-некоторое разбиение множества $X$. Таким образом, топологическое разбиение $[(X, \mathcal{T}), \gamma]$ можно отождествить с тройкой $[X, \mathcal{T}, \gamma]$. В описанной ситуации можно также определить топологию $\mathcal{T}_{\gamma}$ на множестве $\gamma$, называемую фактортопологией на разбиении $\gamma$ (см. [11]).

Ниже вводится ряд свойств топологических разбиений $[(X, \mathcal{T}), \gamma]$ непосредственно в терминах свойств пространства $(X, \mathcal{T})$ и разбиения $\gamma$. Эти свойства не всегда являются свойствами фактортопологии, но тесно связаны с последними.

Приведем несколько определений этого рода. Топологическое разбиение $[(X, \mathcal{T}), \gamma]$ назовем $T_{1}$ разбиением, если каждый элемент $P$ семейства $\gamma$ является замкнутым в пространстве $(X, \mathcal{T})$ множеством. Подмножество $A$ множества $X$ называется $\gamma$-отделенным, или отделенным в топологическом разбиении $z=[(X, \mathcal{T}), \gamma]$, если никакие две точки из $A$ не содержатся в одном элементе семейства $\gamma$.

Подмножество $A \subset X$ называется насыщенным подмножеством топологического разбиения $Z=[(X, \mathcal{T}), \gamma]$, если оно содержит каждый элемент разбиения $\gamma$, с которым пересекается.

Подмножество $A$ множества $X$ называется накрывающим подмножеством топологического разбиения $z=[(X, \mathcal{T}), \gamma]$, если оно пересекается с каждым элементом разбиения $\gamma$. Подмножества $A$ и $B$ множества $X$ называются $\gamma$-подобными (или подобными) если элемент $P$ семейства $\gamma$ пересекается с $A$ в том и только том случае, если $P$ пересекается с $B$.

Топологическое разбиение $[(X, \mathcal{T}), \gamma]$ назовем $\alpha_{1}$-компактным, если существует компактное подпространство $K$ пространства $X$ такое, что $K \cap P \neq \varnothing$, для каждого $P \in \gamma$.

Будем говорить, что топологическое разбиение $[(X, \mathcal{T}), \gamma]$ плотно компактно, если существует накрывающее подмножество $A$ множества $X$, такое что каждое бесконечное подмножество множества $A$ имеет в пространстве $X$ точку полного накопления.

Топологическое разбиение $[(X, \mathcal{T}), \gamma] \alpha_{3}-$ компактно, если для каждого бесконечного $\gamma$-отделенного подмножества $A$ множества $X$ существует $\gamma$-отделенное подмножество $B$ множества $X$, такое что $A$ и $B \gamma$-подобны и у $B$ есть точка полного накопления в $(X, \mathcal{T})$. 
Наконец, $[(X, \mathcal{T}), \gamma]$ фактор-компактно, если фактор-пространство $\left(\gamma, \mathcal{T}_{\gamma}\right)$ компактно. Аналогично, топологическое разбиение $[(X, \mathcal{T}), \gamma]$ назовем фактор-хаусдорфовым, если фактор-пространство $\left(\gamma, \mathcal{T}_{\gamma}\right)$ хаусдорфово. Под мощностью топологического разбиения $[(X, \mathcal{T}), \gamma]$ мы будем понимать мощность семейства $\gamma$.

Следующее утверждение содержит три прозрачных достаточных условия компактности фактор-пространства $\left(\gamma, \mathcal{T}_{\gamma}\right)$.

Предложение 2.1. Если топологическое разбиение $[(X, \mathcal{T}), \gamma] \alpha_{1}$-компактно (плотно компактно, или $\alpha_{3}$-компактно), то фактор-пространство $\left(\gamma, \mathcal{T}_{\gamma}\right)$ компактно (т.е. топологическое разбиение $[(X, \mathcal{T}), \gamma]$ фактор-компактно).

Несложное доказательство этого утверждения для всех трех случаев опускаем.

Представляет интерес и возникающий в связи с понятием топологического разбиения вариант понятия однородности. Напомним, что топологическое пространство называется однородным, если каждую его точку можно перевести в любую точку этого пространства некоторым его гомеоморфизмом на себя. А вот как выглядит аналогичное определение для топологических разбиений. Топологическое разбиение $[(X, \mathcal{T}), \gamma]$ называется однородным, если для любых $P_{1}, P_{2} \in \gamma$ существует гомеоморфизм $g$ пространства $X$ на себя такой, что $g\left(P_{1}\right)=P_{2}$ и $g(P) \in \gamma$, для каждого $P \in \gamma$. Имеет место следующее простое утверждение.

Предложение 2.2. Если топологическое разбиение $[(X, \mathcal{T}), \gamma]$ однородно, то фактор-пространство $\left(\gamma, \mathcal{T}_{\gamma}\right)$ однородно.

Стоит задача выяснить, при каких дополнительных ограничениях верно обратное утверждение. Заслуживает внимания следующий конкретный вопрос о топологических разбиениях: существует ли однородное конечное топологическое разбиение на обычном круге?

Понятие однородного топологического разбиения пространства $(X, \mathcal{T})$ позволяет по-новому определить понятие однородности и для самого пространства $(X, \mathcal{T})$. А именно, скажем, что пространство $(X, \mathcal{T})$ потенииально $\tau$-однородно, где $\tau$ - конечный или бесконечный кардинал, если на $(X, \mathcal{T})$ существует топологическое разбиение $Z=[(X, \mathcal{T}), \gamma]$ мощности $\tau$. Если при этом можно выбрать $\gamma$ вписанным в произвольно заданное конечное открытое покрытие пространства $(X, \mathcal{T})$, то $(X, \mathcal{T})$ назовем вполне $\tau$-однородным.

Топологические разбиения естественно связываются и с понятием размерности. Топологическое разбиение $\mathcal{Z}=[(X, \mathcal{T}), \gamma]$ нульмерно (или ind-нульмерно), если у пространства $(X, \mathcal{T})$ имеется база $\mathcal{B}$, состоящая из множеств, насыщение каждого из которых в $\mathcal{Z}$ замкнуто в пространстве $(X, \mathcal{T})$. При этом, насыщением множества $A \subset X$ в топологическом разбиении $Z=[(X, \mathcal{T}), \gamma]$ называется множество $\bigcup\{P \in \gamma: P \cap A \neq \varnothing\}$.

3. Некоторые результаты. Предположим, что $(G, \mathcal{T})$ - левая топологическая группа и $H-$ некоторая подгруппа группы $G$. Множество $\{x H: x \in G\}$ всех левых косет-множеств $x H$ группы $G$ является дизъюнктным покрытием $\gamma_{H}$ группы $G$ непустыми множествами $x H$. Таким образом, $\left[(G, \mathcal{T}), \gamma_{H}\right]$ является топологическим разбиением. Мы часто обозначаем его через $G_{H}$ и говорим, что оно порождено на $G$ подгруппой $H$.

Когда множество $\{x H: x \in G\}$ всех левых косет-множеств $x H$ левой топологической группы $G$ по подгруппе $H$ берется в качестве множества точек нового топологического пространства, наделенного фактор-топологией (см. [11]), то полученное таким образом пространство называется левым косет-пространством (или левым фактор-пространством) левой топологической группы $G$ по подгруппе $H$ и обозначается $G / H$ (см. [14, теорема 1.5.1]). Естественное отображение множества $G$ на множество $G / H$ обычно обозначается $q$ или $q_{H}$.

Теорема 3.1. Если $G$-линделефова левая топологическая группа счетной тесноты и $H-$ такая замкнутая подгруппа группы $G$, что фактор-пространство $G / H$ компактно и хаусдорфово, то $|G / H| \leqslant 2^{\omega}$.

Доказательство. Так как теснота не увеличивается при факторных отображениях, заключаем, что теснота фактор-пространства $G / H$ счетна. Но пространство $G / H$ однородно, как и всякое 
косет-пространство левой топологической группы (см. [14, теорема 1.5.1]). Теорема де ла Веги (см. [18]) утверждает, что каждый однородный компакт счетной тесноты имеет мощность не большую, чем $2^{\omega}$. Следовательно, $|G / H| \leqslant 2^{\omega}$.

Теорема 3.2. Предположим, что $G$-линделефова левал топологическал группа счетной тесноты и выполняется континуум-гипотеза СН. Предположим также, что $H$ - такая замкнутая подгруппа группы $G$, что левое фактор-пространство $G / H$ компактно и хаусдорфово. Тогда $G / H$ удовлетворяет первой аксиоме счетности.

Доказательство. Имеем: $|G / H| \leqslant 2^{\omega}$ согласно теореме 3.1. Так как косет-пространство $G / H$ компактно и хаусдорфово, из теоремы Чеха-Поспишила следует, что $G / H$ удовлетворяет первой аксиоме счетности хотя бы в одной точке (см. [11]). Из однородности пространства $G / H$ теперь следует, что $G / H$ удовлетворяет первой аксиоме счетности во всех точках.

Проведенное выше рассуждение показывает, что справедливо следующее более общее утверждение. Назовем пространство X FSC-пространством, если каждая свободная последовательность в $X$ счетна. Легко проверяется (и хорошо известно), что каждое линделефово пространство счетной тесноты является $F S C$-пространством (см. [4]). Более того, имеем следующее утверждение.

Предложение 3.3. Если пространство $X$ является обгединением счетного семейства линделефовых пространств счетной тесноты, то X-FSC-пространство.

Доказательство стандартно, и мы его опускаем. Теперь ясно, что справедливы два следующих утверждения.

Теорема 3.4. Если $G$-левая топологическая группа, являющаяся $F S C$-пространством, и $H$ - такая замкнутая подгруппа группы $G$, что фактор-пространство $G / H$ компактно и хаусдорфово, то $|G / H| \leqslant 2^{\omega}$.

Теорема 3.5. Предположсим, что $G$ - левая топологическая группа, являющаяся FSC-пространством, и что выполняется континуум-гипотеза $C H$. Предположим также, что $H$ такая замкнутая подгруппа группь $G$, что фактор-пространство $G / H$ компактно и хаусдорфово. Тогда $G / H$ удовлетворлет первой аксиоме счетности.

4. Полные по Чеху группы и их образы. Полученные выше результаты могут быть применены к исследованию непрерывных образов полных по Чеху топологических групп.

Предложение 4.1. Предположим, что $G$ - левая топологическая группа, являющаяся полным по Чеху пространством, и что $H$ - замкнутая подгруппа группы $G$, для которой левое косет-пространство $G / H$ хаусдорфово. Тогда $G / H$ компактно в том и только том случае, если существует такое компактное подпространство $K$ пространства $G$, что $q(K)=G / H$, где $q$ - фактор-отображение топологической группы $G$ на левое фактор-пространство $G / H$.

Доказательство. Если такой компакт $K$ существует, то $G / H$ компактно, так как $q(K)=G / H$ и $q$ непрерывно.

Предположим теперь, что $G / H$ компактно. Тогда из фактов, что отображение $q: G \rightarrow G / H$ открыто и непрерывно, а пространство $G$ полно по Чеху, следует, что существует такое компактное подпространство $K$ пространства $G$, что $q(K)=G / H$ (см. [3, теорема 1.2].

Следствие 4.2. Предположим, что $G$ - полная по Чеху левая топологическая группа и $H-$ замкнутая подгруппа группы $G$, для которой левое косет-пространство $G / H$ хаусдорфово. Тогда следующие условия попарно равносильны:

(1) фактор-пространство $G / H$ компактно;

(2) топологическое разбиение $\left[(G, \mathcal{T}), \gamma_{H}\right]$, где $\gamma_{H}=\{x H: x \in G\}, \alpha_{3}$-компактно;

(3) топологическое разбиение $\left[(G, \mathcal{T}), \gamma_{H}\right]$, где $\gamma_{H}=\{x H: x \in G\}$, плотно компактно;

(4) топологическое разбиение $\left[(G, \mathcal{T}), \gamma_{H}\right]$, где $\gamma_{H}=\{x H: x \in G\}, \alpha_{1}$-компактно. 
Следствие вытекает из предложения 2.1 и предложения 4.1.

При изучении топологических разбиений нет никакой необходимости ограничиваться топологическими группами и их косет-пространствами. Заметим, что каждое (сюръективное) отображение $f$ топологического пространства $X$ на множество $Y$ порождает топологическое разбиение $\gamma_{f}=\left\{f^{-1}(y): y \in Y\right\}$ пространства $X$ на прообразы точек из $Y$ при $f$. Это разбиение можно обозначить символом $[X, \mathcal{T}, f]$, где $\mathcal{T}$ - топология на $X$.

Теорема 4.3. Предположсим, что $G$ - полное по Чеху подпространство топологической группы $M$, являющееся множеством типа $G_{\delta}$ в $M, u$ что $f$-непрерывное открытое отображение пространства $G$ на хаусдорфово пространство $Y$. Тогда произвольный компакт $F$, лежащий в $Y$, содержится в некотором диадическом компакте $F_{1} \subset Y . B$ частности, если само пространство $Y$ является компактом, то этот компакт диадический.

Доказательство. Как известно, каждое непрерывное открытое отображение $f$ полного по Чеху пространства $X$ на хаусдорфово пространство $Y$ является компактно накрывающим, т.е. для каждого компакта $P \subset Y$ существует такой компакт $B \subset X$, что $f(B)=P($ см. [3, теорема 1.2]). Отсюда вытекает, что в условиях теоремы 4.3 найдется компакт $K$, лежащий в $G$, для которого $f(K)=F$. Но $G$ является пространством счетного типа, так как $G$ полно по Чеху (см. [2]). Следовательно, найдется такой компакт $K_{0} \subset G$ типа $G_{\delta}$ в $G$, что $K \subset K_{0}$. Так как $G$ является множеством типа $G_{\delta}$ в $M$, а $K_{0}$ - множество типа $G_{\delta}$ в $G$, видим, что $K_{0}$ является множеством типа $G_{\delta}$ в топологической группе $M$. Ясно, что $F \subset f\left(K_{0}\right)$. Как показал М. М. Чобан (см. [9]), каждый компакт типа $G_{\delta}$ в топологической группе является диадическим. Следовательно, $K_{0}-$ диадический компакт. Тогда и $f\left(K_{0}\right)$ - диадический компакт. Остается положить $F_{1}=f\left(K_{0}\right)$.

Следующее утверждение близко к приведенному выше результату.

Теорема 4.4. Предположим, что $(G, \mathcal{T})$ - топологическал группа и подпространство $P \subset G$ является множеством типа $G_{\delta}$ в $(G, \mathcal{T})$ и перистым пространством. Предположим также, что хаусдорфово пространство $Y$ является непрерывным образом пространства $P$ и компакт $F \subset Y$ является образом при $f$ компакта $K \subset P$. Тогда существует такой диадический комnaкm $F_{0} \subset Y$, ито $F \subset F_{0}$.

Доказательство теоремы 4.4 близко к доказательству теоремы 4.3. Из теоремы 4.3 легко получается следующее известное утверждение (см. [9]).

Следствие 4.5. Предположим, что $G$ - полная по Чеху топологическая группа и $H$-замкнутая подгруппа группь $G$, для которой левое фактор-пространство $G / H$ является компактным пространством. Тогда $G / H$-диадический компакт, а фактор-отображение $q: G \rightarrow$ $G / H$ является компактно накрывающим.

Действительно, в [9] показано, что каждое компактное косет-пространство полной по Чеху топологической группы представимо и как косет-пространство $\omega$-узкой топологической группы. В. В. Успенский показал (см. [7]), что если $\omega$-узкая топологическая группа непрерывно и транзитивно действует на некотором компакте $F$, то $F$ является компактом Дугунджи.

Следствие 4.6. Предположим, что $G$ - полное по Чеху подпространство топологической группы $M$, являющееся множеством типа $G_{\delta}$ в $M$, и что $f$-непрерывное открытое отображение пространства $G$ на хаусдорфово пространство $Y$ счетной тесноты. Тогда произвольный компакт $F$, лежсащий в $Y$, метризуем. В частности, если само пространство $Y$ является компактом, то этот компакт $Y$ метризуем.

Доказательство. По теореме 4.3 компакт $F$ содержится в некотором диадическом компакте $F_{1} \subset$ $Y$. Теснота компакта $F_{1}$ счетна, так как она не превосходит тесноты пространства $Y$. Остается сослаться на хорошо известный факт: каждый диадический компакт счетной тесноты метризуем (см. [5]). Заключаем, что компакт $F_{1}$ метризуем и, следовательно, компакт $F$ метризуем. 
В частности, последнее утверждение применимо к полным по Чеху топологическим группам. В связи с последним результатом естественно ввести следующее понятие. Топологическое пространство $X$ называется $G_{\delta}$-окруженным, если существует топологическая группа $G$, содержащая $X$ как подпространство, являющееся множеством типа $G_{\delta}$ в $G$.

В [12] показано, что если $G$-топологическая группа, а $f$-непрерывное отображение пространства $G$ на хаусдорфово пространство $F$ с первой аксиомой счетности, причем $f(K)=F$, для некоторого компактного подпространства $K$ пространства $G$, то $F$ сепарабельно и метризуемо. Это утверждение обобщает классическую теорему А. С. Есенина-Вольпина (см. [6, 11]), согласно которой каждый диадический компакт $F$ с первой аксиомой счетности метризуем. Однако следующие вопросы, насколько мне известно, остаются открытыми.

Проблема 4.7. Предположим, что $G$-линделефова топологическая группа счетной тесноты и $F$-компакт с первой аксиомой счетности, являющийся непрерывным образом пространства $G$. Верно ли тогда, что компакт $F$ метризуем?

Проблема 4.8. ([12]). Предположим, что $G$ - топологическая группа и $K$-ее компактное подпространство. Предположим также, что $f$-непрерывное отображение пространства $G$ на компакт $F$, для которого $f(K)=F$. Верно ли тогда, что компакт $F$ диадичен?

Стоит отметить, что теорема 4.3 и следствие 4.6 частично распространяются и на непрерывные замкнутые отображения.

Теорема 4.9. Предположим, что $G$ - полная по Чеху топологическая группа и $f$-непрерывное замкнутое отображение пространства $G$ на хаусдорфово пространство $Y$. Тогда произвольный компакт $F$, лежащий в $Y$, содержится в некотором диадическом компакте $F_{1} \subset Y$. В частности, если само пространство $Y$ является компактом, то этот компакт диадический.

Доказательство. Известно, что каждая полная по Чеху топологическая группа паракомпактна (см. [14]). Э. Майкл (E. Michael) показал, что каждое непрерывное замкнутое отображение $f$ паракомпактного хаусдорфова пространства $X$ на любое топологическое пространство $Y$ является компактно накрывающим (см. [16]). Следовательно, имеет место следующее утверждение.

Предложение 4.10. Каждое непрерывное замкнутое отображение полной по Чеху топологической группы на произвольное пространство является компактно накрывающим.

Отсюда вытекает, что найдется компакт $K$, лежащий в $G$, для которого $f(K)=F$. Далее рассуждаем, как при доказательстве теоремы 4.3.

Теорема 4.11. Предположим, что $G$ - полная по Чеху топологическая группа и $f$-непрерывное замкнутое отображсение пространства $G$ на хаусдорфово пространство $Y$ счетной тесноты. Тогда произвольный компакт $F$, лежащий в $Y$, метризуем. В частности, если само пространство $Y$ является компактом, то этот компакт метризуем.

\section{СПИСОК ЛИТЕРАТУРЫ}

1. Архангельский А. В. Факторные отображения метрических пространств// Докл. АН СССР. - 1964. - 155. - C. $247-250$.

2. Архангельский A. В. Бикомпактные множества и топология пространств// Тр. Моск. мат. о-ва. 1965. - 13. - C. $3-55$.

3. Архангельский A. В. Открытые и близкие к ним отображения. Связи между пространствами// Тр. Моск. мат. о-ва. - 1966. - 15. - С. 181-223.

4. Архангельский A. B. Топологическая однородность. Топологические группы и их непрерывные образы// Усп. мат. наук. - 1987. - 42, № 2. - С. 69-105.

5. Архангельский А. В., Пономарев В. И. О диадических бикомпактах// Докл. АН СССР. - 1968. 182. - C. 993-996.

6. Есенин-Вольпин A. С. О соотношении между локальным и интегральным весом в диадических бикомпактах// Докл. АН СССР. - 1949. - 68. - С. 441-444. 
7. Успенский В. В. Топологические группы и компакты Дугунджи// Мат. сб. - 1989. - 180, № 8. C. $1092-1118$.

8. Успенский В. В. Компактные факторпространства топологических групп и спектры Хейдона// Мат. заметки. - 1987. - 42, № 4. - С. 594-602.

9. Чобан M. M. Топологическое строение подмножеств топологических групп и их фактор-пространств// в кн.: Топологические структуры и алгебраические системы. - Кишинев: Штиинца, 1977. C. $117-163$.

10. Чобан M. М. Редукционные теоремы о существовании неперывных сечений// в кн.: Сечения над подмножествами факторпространств топологических групп. - Кишинев: Штиинца, 1973. - С. 111-156.

11. Энгелькинг Р. Общая топология. - М.: Мир, 1986.

12. Arhangel'skii A. V. Generalizing dyadicity and Esenin-Vol'pin's theorem// Topol. Appl. (to appear).

13. Arhangel'skii A. V., van Mill J. Topological homogeneity// in: Recent Progress in General Topology, III (Hart K. P., van Mill J., Simon P., eds.). - Atlantis Press, 2014. - P. 1- 68.

14. Arhangel'skii A. V., Tkachenko M. G. Topological Groups and Related Structures. — Paris: Atlantis Press/World Scientific, 2008.

15. Michael E. A theorem on semi-continuous set-valued functions// Duke Math. J. — 1959. — 26. — P. 647651.

16. Michael E. A note on closed maps and compact sets// Isr. J. Math. — 1964. — 2. — P. 173-176.

17. van Mill J. Homogeneous spaces and transitive actions by Polish groups// Isr. J. Math. — 2008. — 165 (1). - P. 133-159.

18. de la Vega R. A new bound on the cardinality of homogeneous compacta// Topol. Appl. — 2006. — 153. - P. $2118-2123$.

Архангельский Александр Владимирович

Московский педагогический государственный университет

E-mail: arhangel.alex@gmail.com 